\title{
Platinum (II) Nanofibers as Assembly-driven Inhibitors of Metabolic Adaptation in Cancer Cells
}

Zhixuan Zhou', Konrad Maxeiner ${ }^{1}$, Pierpaolo Moscariello', Siyuan Xiang ${ }^{1}$, Yingke Wu${ }^{1}$, Colette J. Whitfield ${ }^{1}$, Lujuan Xu${ }^{1}$, Anke Kaltbeitzel ${ }^{1}$, Shen Han ${ }^{1}$, David Mücke ${ }^{2}$, Haoyuan Qi²,3 Manfred Wagner ${ }^{1}$, Ute Kaiser ${ }^{2}$, Katharina Landfester ${ }^{1}$, Ingo Lieberwirth ${ }^{1}$, David Y.W. Ng ${ }^{1, *}$, Tanja Weil ${ }^{1, *}$

${ }^{1}$ Max Planck Institute for Polymer Research, 55128 Mainz, Germany.

${ }^{2}$ Central Facility of Materials Science Electron Microscopy, Universität Ulm, 89081 Ulm, Germany.

${ }^{3}$ Faculty of Chemistry and Food Chemistry \& Center for Advancing Electronics Dresden (cfaed), Technische Universität Dresden, 01062 Dresden, Germany.

\begin{abstract}
Nanostructure-based functions are omnipresent in biology and essential for the diversity of life. Despite their importance, it is difficult to establish mechanisms that define their bioactivity and rationalize them through synthetic designs. As such, strategies that connect bioactive functions through structure formation are scarce. Herein, we design a near-infrared emitting platinum (II)tripeptide that undergoes a rearrangement using endogenous $\mathrm{H}_{2} \mathrm{O}_{2}$ to rapidly assemble into fibrillar superstructures. The resultant assembly inhibits the metabolism of aggressive metastatic MDA-MB231 cells and A549 cells at the systemic level by blocking aerobic glycolysis and oxidative phosphorylation, thereby shutting down ATP production. Hence, ATP-dependent actin formation and glucose metabolite-dependent histone deacetylase activity are downregulated, leading to apoptosis. By demonstrating that assembly-driven functions can inhibit broad biological pathways, supramolecular nanostructures could offer the next generation biomedical solutions beyond conventional applications.
\end{abstract}

\section{Introduction}

In cells, protein nanostructures are abundant, serving as cellular scaffolds, multi-domain catalysts, and transport highways ${ }^{1}$. Among them, ordered protein assemblies consisting of $\beta$-sheet structures, so-called $\beta$-amyloids, play both functional and pathological roles. They serve as biotemplates, within melanocytes, for melanin biosynthesis ${ }^{2,3}$ or as nucleating centers towards the amyloidosis in Type 2 diabetes $^{4,5}$. Mechanistically, the monomers of these ordered nanostructures do not possess intrinsic functions of their own, but rely on the propagation of long-range hierarchical structures to feature their rich biological activities.

Several strategies have since attempted to re-engineer these nanostructures using simplified peptidebased monomers to elicit biomedical functions ${ }^{6-13}$. Pharmacologically, self-assembling nanostructures in cells combine features of small molecules such as deep cell/tissue penetration together with properties from larger superstructures like enzymatic stability and retardation of cellular efflux ${ }^{10,14,15}$. However, in contrast to molecular drugs and prominent biologics, such as antibodies or mRNA, which target a specific enzyme, receptor or gene expression, very little is known on the biochemical profile of intracellularly self-assembled nanostructures. We rationalized that nanostructures could plausibly exhibit broad bioactivity on a systemic level that impacts multiple biological pathways simultaneously. 
In this regard, metabolism is a critical set of processes that define cellular life, and it has witnessed increasing focus due to the rising significance of aerobic glycolysis (AGlyc) in cancer cells ${ }^{16-18}$. Also termed as the Warburg effect, AGlyc upregulates the processing of glucose to produce ATP in the presence of oxygen while paradoxically competing against a more efficient oxidative phosphorylation pathway (OxPhos) ${ }^{19}$. The preference for AGlyc aims to conserve anabolic carbon resources for biosynthesis required for cancer cell proliferation, whereas OxPhos breaks these resources down into $\mathrm{CO}_{2}$ that exits cellular metabolism ${ }^{10}$. Inhibitors of AGlyc or OxPhos have recently shown great promise in addressing aggressive triple-negative MDA-MB-231 breast cancer and A549 lung cancer, which have a poor prognosis as effective therapies are currently lacking ${ }^{20,21}$. Particularly in the metastatic state when cells can dynamically switch between AGlyc/OxPhos metabolism with increased drug efflux, treatment regimens with chemotherapeutics, such as cisplatin, are largely ineffective, resulting in resistances towards chemotherapies over time ${ }^{22}$.

We propose that cancer cell adaptation can be broadly targeted by assembly-driven metabolic inhibition, by blocking AGlyc/OxPhos pathways and thus ATP-dependent downstream processes. The assembly-driven metabolic inhibitor (ADMI) reported herein comprises a platinum (II) terpyridine (Pt-tpy), which serves as a near-infrared (NIR) motif promoting $\pi-\pi$ interactions and intermolecular metal-metal alignment into ordered $\beta$-amyloid-like structures ${ }^{23,24}$. Tethered to the Pt-tpy is an isotripeptide, where its isomerization between the "kinked" monomeric and linear self-assembling forms is controlled by elevated levels of reactive oxygen species (ROS) (Fig. 1a) ${ }^{11}$. The structural transition into the ADMI is assessed on triple-negative MDA-MB-231 cells, derived from a highly metastatic cancer, and A549 cells. The impact of the ADMI on AGlyc/OxPhos leading to histone deacetylase (HDAC) activity, actin restructuring, and apoptosis is investigated. We demonstrate that assemblydriven functions can be leveraged as an alternative treatment modality where supramolecular structures modulate bioactivity in a complex living environment. 
a

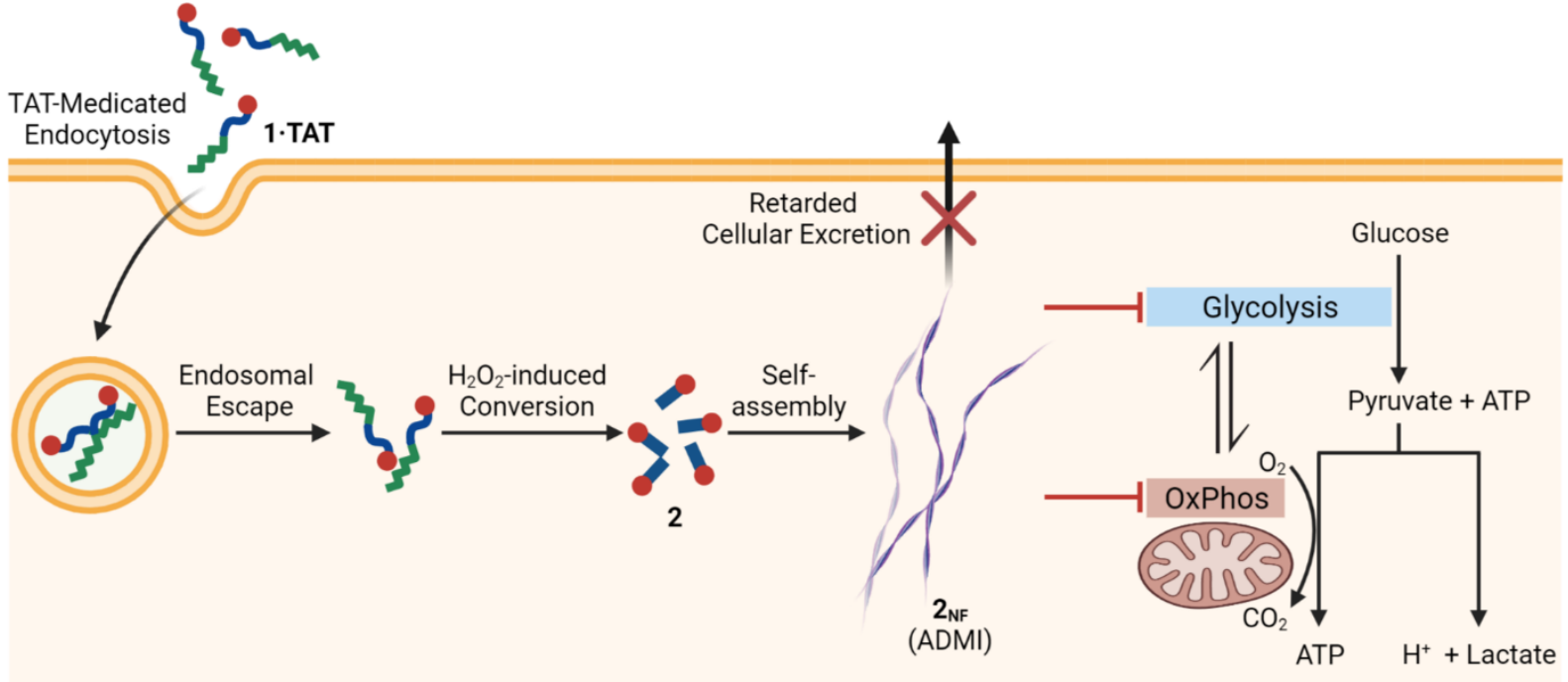

b

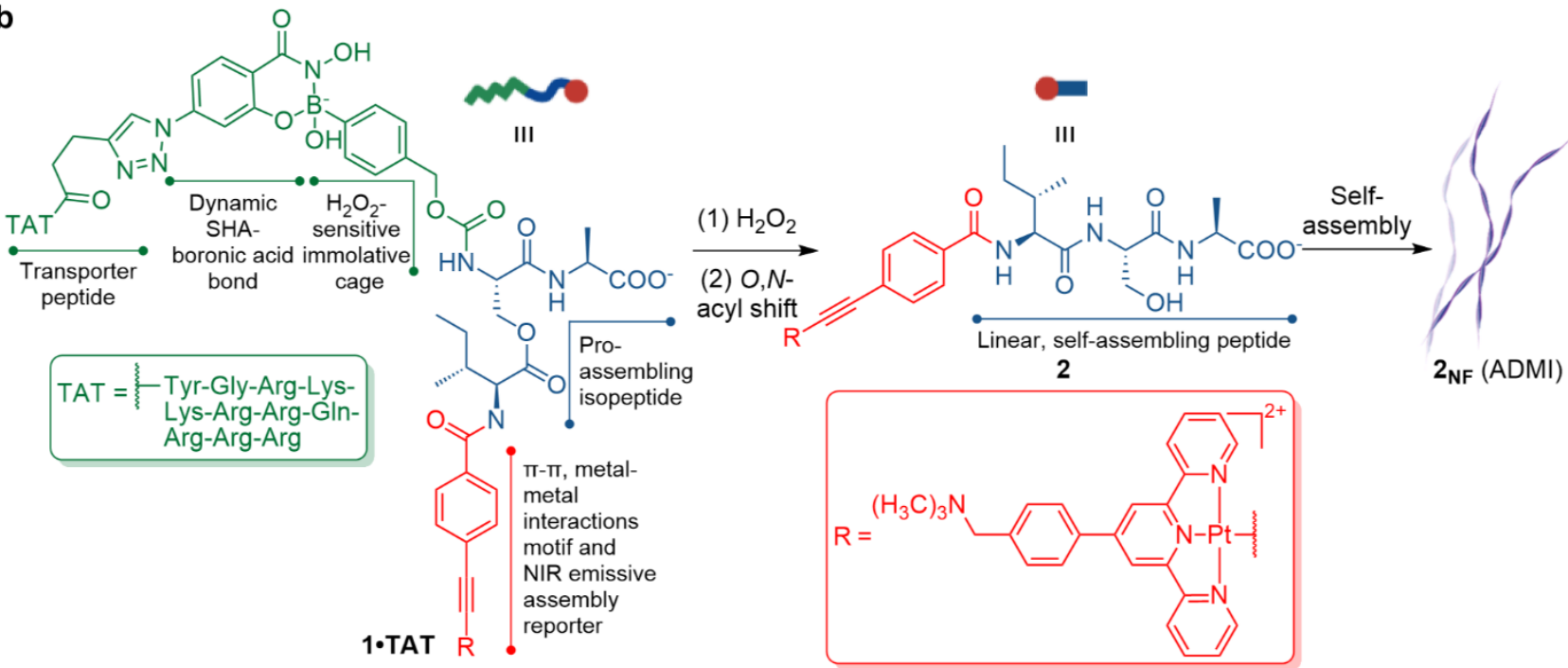

Fig. 1| Intracellular assembly of platinum (II)-containing nanofibers can disrupt aerobic cancer cell metabolism and cause cancer cell apoptosis. a, Schematic illustration of the intracellular $\mathrm{H}_{2} \mathrm{O}_{2}$-induced formation of linear platinum (II) complexes that self-assemble into nanofibers, which inhibit cell metabolism. $\mathbf{b}$, Chemical design for the trans-activator of transcription (TAT) peptide appended isopeptide-platinum (II) complex 1-TAT and its reaction scheme for intracellular $\mathrm{H}_{2} \mathrm{O}_{2}$-induced conversion to linear complex 2, which then undergoes self-assembly in the cytosol to form nanofibers $\mathbf{2}_{\mathrm{NF}}$.

\section{Design and $\mathrm{H}_{2} \mathrm{O}_{2}$ cascade transformation of ADMI precursors}

The isopeptide-platinum (II) complex 1-TAT is composed of three functional modules (Fig. 1b): 1) a pro-assembling isopeptide (ISA) caged by an immolative boronic acid group ${ }^{11}$, 2) a transporter peptide (trans-activator of transcription, TAT) ${ }^{25}$ connected to the boronic acid group via a dynamic covalent salicylhydroxamate (SHA)-boronic acid bond ${ }^{26}$, and 3) a Pt-tpy complex coordinated to the alkynyl group on the $\mathrm{N}$-terminus of the ISA peptide. The expected mechanism of how the complex interacts with cells would begin with the TAT-mediated endocytosis and intracellular endosomal escape of the complex ${ }^{11}$. Only at near-neutral ( $\mathrm{pH}$ 7.4) cytosolic conditions, endogenous $\mathrm{H}_{2} \mathrm{O}_{2}$ possess sufficient oxidative strength to immolate the boronic acid cage ${ }^{11}$. The exposed $-\mathrm{NH}_{2}$ of the serine residue 
undergoes an $\mathrm{O}, \mathrm{N}$-acyl shift, causing the peptide to rapidly isomerize into the monomeric assembly precursor 2 (Supplementary Fig. 27), which assembles into the ADMI nanofibers $\mathbf{2}_{\mathrm{NF}}$.

The kinetics of the $\mathrm{H}_{2} \mathrm{O}_{2}$-triggered formation of 2 in solution was investigated by high performance liquid chromatography-mass spectrometry (LC-MS) (Fig. 2a-d, Supplementary Fig. 28). In aqueous solution, incubation with $\mathrm{H}_{2} \mathrm{O}_{2}$ for 20 min resulted in the complete disappearance of 1 and the emergence of two new peaks with retention times $\left(t_{R}\right)$ of $5.69 \mathrm{~min}$ and $6.06 \mathrm{~min}$, respectively (Fig. 2b) both showing $\mathrm{m} / \mathrm{z}$ values in agreement with the chemical formula of $2\left(\mathrm{~m} / \mathrm{z}=496.4\right.$ for $[2+\mathrm{H}]^{+}$and 991.3 for $\left.[2]^{+}\right)$(Fig. 2c, Supplementary Fig. 29). In comparison with 2 obtained via synthesis $\left(t_{\mathrm{R}}=6.06\right.$ $\mathrm{min}$ ), the former was assigned to be the intermediate formed (iso-2) upon the immolation of the phenylboronic acid prior to isomerization. Further incubation with $\mathrm{H}_{2} \mathrm{O}_{2}$ led to an increase of 2, with a 95\% conversion at $4 \mathrm{~h}$ (Fig. $2 \mathrm{~d}$ ). In contrast, less than 5\% conversion was observed after $24 \mathrm{~h}$ incubation of 1 without $\mathrm{H}_{2} \mathrm{O}_{2}$ (Supplementary Fig. 30).
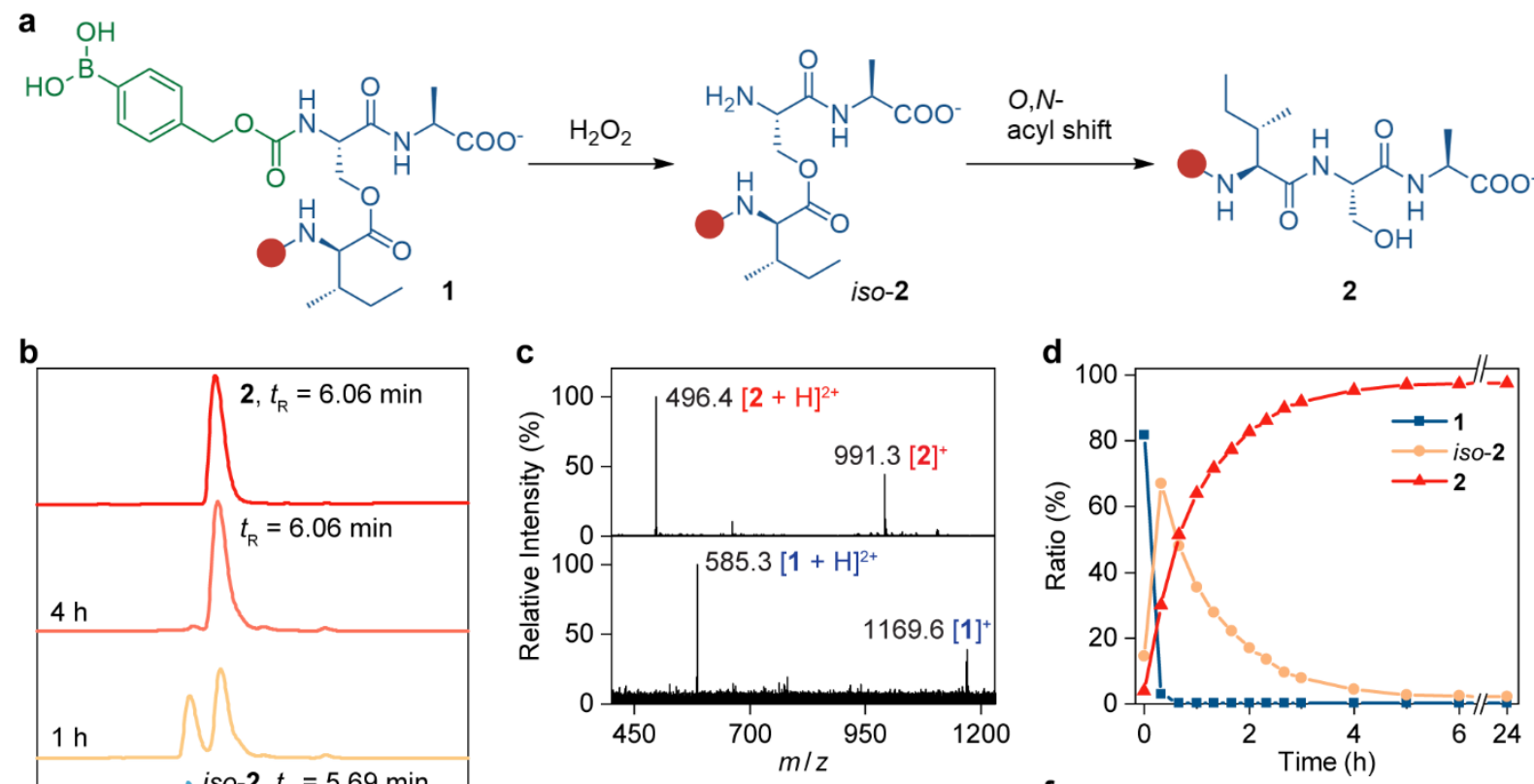

b

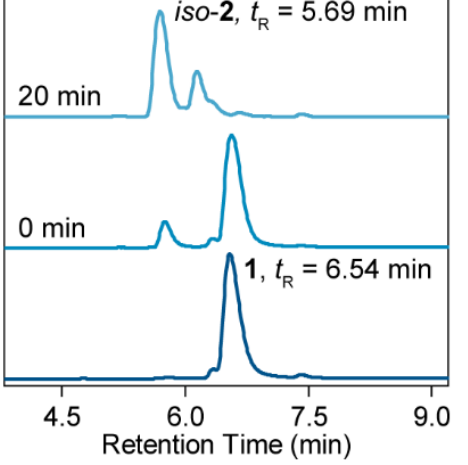

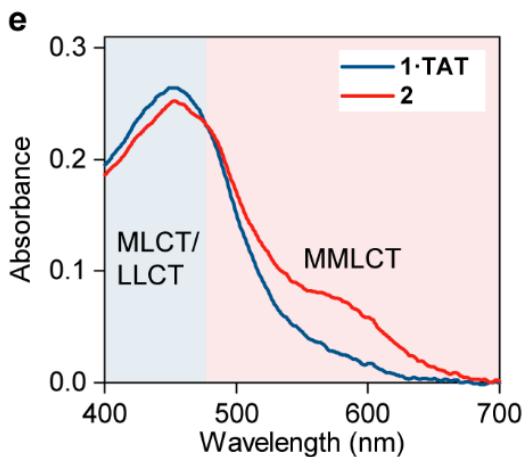
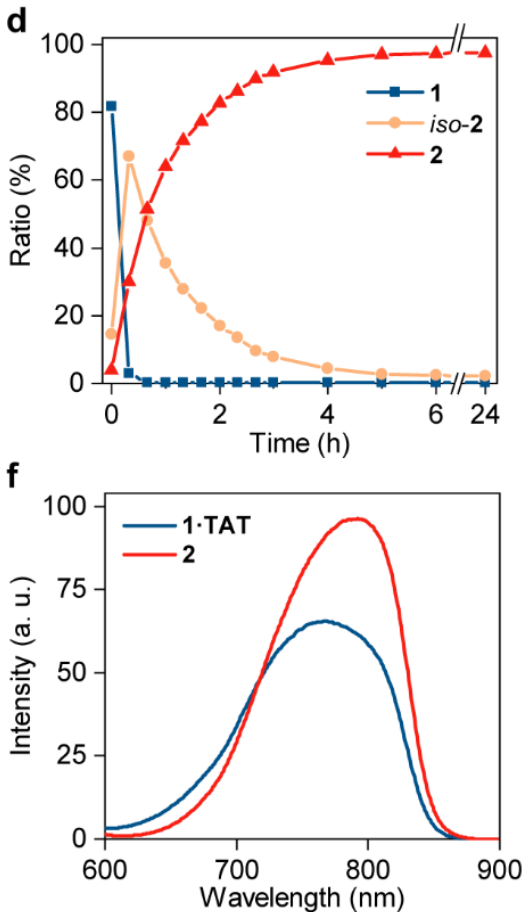

Fig. 2| $\mathrm{H}_{2} \mathrm{O}_{2}$-induced formation of 2 in PB. a, Reaction scheme for the conversion of 1 to 2 in $\mathrm{H}_{2} \mathrm{O}_{2}$ in nearneutral $\mathrm{pH} . \mathrm{H}_{2} \mathrm{O}_{2}$ removes the phenylboronic acid cage, leading to the formation of intermediate iso-2 with free $-\mathrm{NH}_{2}$. Subsequent $\mathrm{O}, \mathrm{N}$-acyl rearrangement of the isopeptide bond in iso-2 generates 2. b, LC-MS kinetic analysis over the $\mathrm{H}_{2} \mathrm{O}_{2}(0.5 \mathrm{mM})$-induced linearization of $1(50 \mu \mathrm{M})$ in a mixture of $\mathrm{NH}_{4} \mathrm{HCO}_{3}$ buffer $(\mathrm{pH} 7.4,20$ $\mathrm{mM}$ ) and $\mathrm{CH}_{3} \mathrm{OH}(9 / 1$, vol\%). c, Convoluted MS spectra in the LC-MS analysis for a reaction time of 0 min and $t_{R}=6.06-6.21 \mathrm{~s}$ (identified as complex 1 ) and a reaction time of $4 \mathrm{~h}$ and $t_{\mathrm{R}}=6.54-6.69 \mathrm{~s}$ (identified as complex 2). d, Molar ratio of 1, iso-2 and 2 after addition of $\mathrm{H}_{2} \mathrm{O}_{2}$ based on the peak integration at $254 \mathrm{~nm}$. e, UV-Vis 
absorption spectra of 1.TAT and $2(50 \mu \mathrm{M})$ in PB (pH 7.4, $50 \mathrm{mM})$. $\mathbf{f}$, Luminescence emission spectra of 1.TAT and $2(50 \mu \mathrm{M})$ in $\mathrm{PB}(\mathrm{pH} 7.4,50 \mathrm{mM})$. Excitation wavelength $=488 \mathrm{~nm}$.

The square-planar platinum (II) center in the complexes endows the ADMI with optical properties associated with the nature of self-assembly. Complexes 1-TAT and 2 displayed absorption bands centered at $460 \mathrm{~nm}$ in PB (Fig. 2e), which can be assigned to a combination of $d \pi(P t) \rightarrow \pi^{*}($ tpy) metal-to-ligand charge-transfer (MLCT) and alkynyl-to-tpy ligand-to-ligand charge-transfer (LLCT) transitions. A lower energy absorption shoulder at ca. $570 \mathrm{~nm}$ is observed for both complexes, which can be attributed to a metal-metal-to-ligand charge-transfer (MMLCT) transition ${ }^{23,27}$. Complex 2 exhibited weaker MLCT/LLCT transition but markedly stronger MMLCT absorption than 1-TAT. This indicates a higher extend of intermolecular $d_{z}^{2}$ interactions between platinum (II) centers in neighboring molecules of $\mathbf{2}$, suggesting a proclivity for self-assembly in PB forming $2 \mathrm{NF}^{23,27}$. Both solutions showed NIR emission originating from the MMLCT excited states upon excitation at $488 \mathrm{~nm}$ (Fig. 2f). Complex 1-TAT exhibited emission centered at $770 \mathrm{~nm}$, whereas complex 2 displayed redshifted emission $\left(\lambda_{\max }=792 \mathrm{~nm}\right)$ with a 1.5 -fold higher intensity, which can be ascribed to the enhanced MMLCT transition as a result of self-assembly. The difference in the photophysical profile between the solutions of the complexes facilitate the tracking of their $\mathrm{H}_{2} \mathrm{O}_{2}$-induced conversion and subsequent assembly of 2 into $2 \mathrm{NF}$ in PB. Treatment of 1-TAT with $\mathrm{H}_{2} \mathrm{O}_{2}(0.5 \mathrm{mM})$ led to a red-shift in the $\lambda_{\max }$ with a concomitant increase in emission intensity (Supplementary Fig. 31). The conversion is completed in $\mathbf{2 8 0}$ min with the final emission profile resembling that of $\mathbf{2} \mathrm{NF}$, in agreement with the LCMS study.

\section{ADMI nanofibers $2_{\mathrm{NF}}$ show high molecular order.}

The self-assembly of the complexes were visualized using transmission electron microscopy (TEM). Nanofibers were observed for $\mathbf{2}_{\mathrm{NF}}$ at concentrations ranging from 5 to $100 \mu \mathrm{M}$ (Fig. 3a, Supplementary Fig. 32), whereas 1 and 1.TAT showed no defined nanostructures (Supplementary Fig. 33). Upon oxidation of 1-TAT by $\mathrm{H}_{2} \mathrm{O}_{2}$, the transformation into 2 and subsequent self-assembly produced similar fibrous morphology to the control (Supplementary Fig. 34). Selected-area electron diffraction on $2_{\mathrm{NF}}$ showed diffraction arcs with $3.3 \AA$ lattice spacing, in agreement with the intermolecular distance of $\pi$ $\pi$ interactions within the nanofibers ${ }^{28}$. The diffraction arcs are perpendicular to the long axis of the fibers (Fig. 3b, Supplementary Fig. 35). Nanofiber formation is further supported by cryogenic highresolution TEM studies on $\mathbf{2}_{\mathrm{NF}}$ in $\mathrm{PB}$, where the growth axis suggested an end-to-end molecular arrangement of the complexes (Fig. 3c, Supplementary Fig. 36). Each fiber was observed to be bundled with a mean interfiber distance of $2.9 \mathrm{~nm}$.

Circular dichroism (CD) spectroscopy analysis on $\mathbf{2}_{\mathrm{NF}}$ revealed a maximum at $190 \mathrm{~nm}$ corresponding to the $\Pi \rightarrow \pi^{*}$ of the carbonyl group, depicting $\mathrm{H}$-bond interactions centered on the peptidic backbone. This is coupled with the red-shifted $n \rightarrow \pi^{*}$ transition visible at the $229 \mathrm{~nm}$ minimum ${ }^{29}$. Exciton coupling parallel to the peptide backbone also exists for $2_{\mathrm{NF}}$ at $210 \mathrm{~nm}^{29}$ (Fig. $3 \mathrm{~d}$ ). Additionally, a strong positive signal beyond $250 \mathrm{~nm}$ corresponding to the $\pi \rightarrow \pi^{*}$ transition of the Pt-tpy group ${ }^{23}$ is also observed for $\mathbf{2}_{\mathrm{NF}}$, demonstrating the importance of the Pt-tpy group in the self-assembly. In contrast, $\mathbf{1}$ only exhibited a minimum at $208 \mathrm{~nm}$ (Supplementary Fig. 37). The self-assembly behavior of $\mathbf{2}_{\mathrm{NF}}$ at the 
molecular level was further examined using NMR. At $298 \mathrm{~K}, 2_{\mathrm{NF}}$ showed broad signals in the ${ }^{1} \mathrm{H}$ NMR spectrum. Increasing the temperature led to sharping of the signals accompanied by significant downfield shifts (Supplementary Fig. 39), which indicates self-assembly at 298 K. Nuclear Overhauser effect spectroscopy (NOESY) of $\mathbf{2}_{\mathrm{NF}}$ revealed NOE cross peaks between the protons on the tpy group and the phenyl ring of the alkynyl ligand $\left(H^{\mathrm{d}} / H^{\mathrm{n}}\right.$ and $\left.H^{\mathrm{e}} / H^{\mathrm{h}}\right)$, and between the non-neighboring protons on the tpy moiety $\left(H^{\mathrm{a}} / H^{\mathrm{c}}, H^{\mathrm{p}} / H^{\mathrm{d}}\right.$, and $\left.H^{\mathrm{p}} / H^{\mathrm{e}}\right)$ (Fig. 3e, Supplementary Fig. 45), implying that the Pt-tpy group adopt a twisted head-to-tail stacking upon self-assembly (Fig. 3f), which is characteristic for Pttpy complexes ${ }^{27}$. The microscopy and spectroscopy experiments suggest that $\mathbf{2}_{\mathrm{NF}}$ possesses a high degree of molecular order, which is highly favorable for studies in a biological context because it enhances the proteolytic stability of the nanofibers and promotes NIR emission originated from the Pt-tpy group (Fig. 3g).

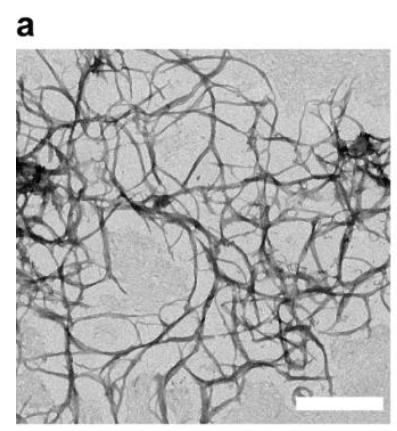

b

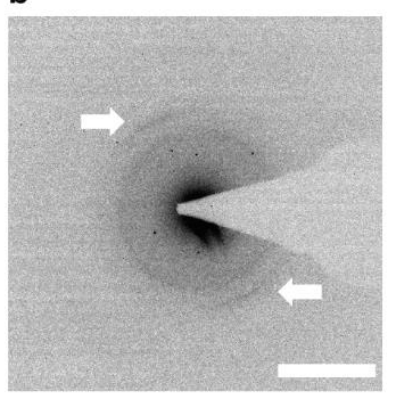

c

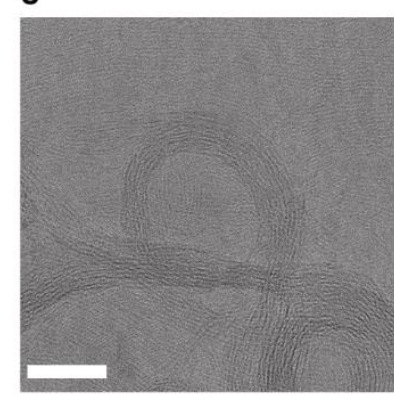

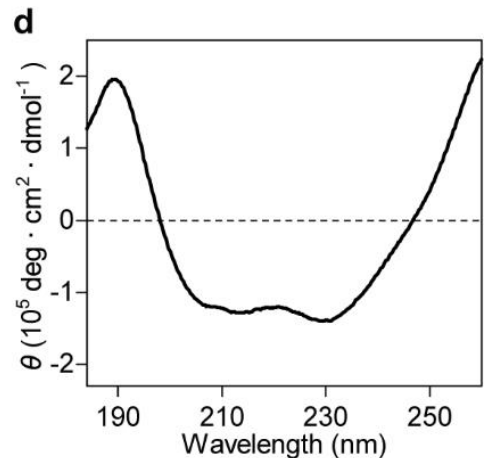

e

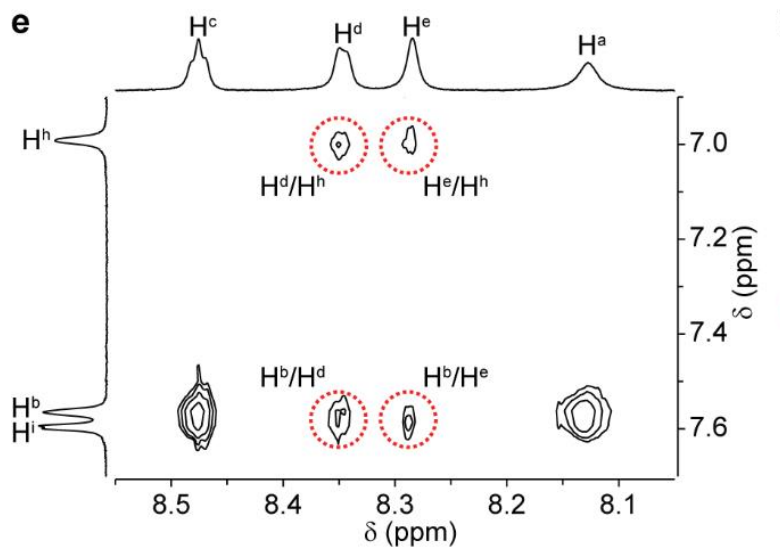

f

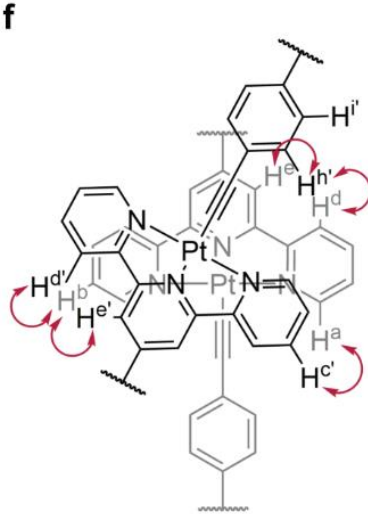

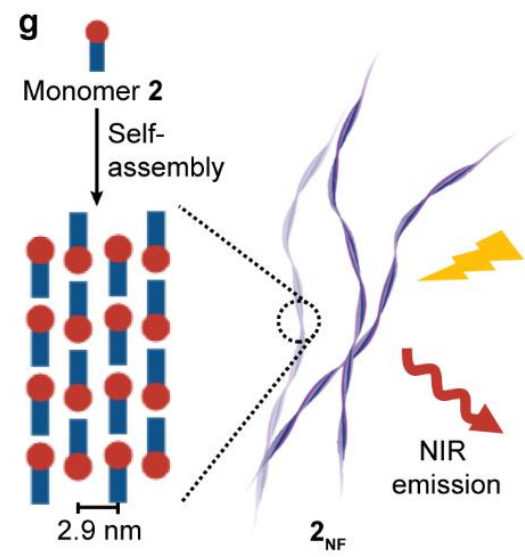

Fig. 3| Nanofiber $2_{\mathrm{NF}}$ with a high degree of molecular order formed by self-assembly of 2. a, TEM image of the nanofibers $22_{\mathrm{NF}}(25 \mu \mathrm{M})$ in $\mathrm{PB}(\mathrm{pH} 7.4,10 \mathrm{mM})$. Scale bar, $200 \mathrm{~nm}$. b. Selected-area electron diffraction pattern of the nanofiber $2 \mathrm{NF}(100 \mu \mathrm{M})$ in $\mathrm{H}_{2} \mathrm{O}$. The white arrows indicate diffraction arcs at $3.0 \mathrm{~nm}^{-1}$, i.e., $3.3 \AA$. Scale bar, $3 \mathrm{~nm}^{-1}$. c, Cryogenic high-resolution TEM image of the nanofiber $\mathbf{2}_{\mathrm{NF}}(25 \mu \mathrm{M})$ in $\mathrm{PB}(\mathrm{pH} 7.4,10 \mathrm{mM})$. Scale bar, $50 \mathrm{~nm}$. d, Circular dichroism (CD) spectra of $22_{\mathrm{NF}}$ in a mixture of $\mathrm{PB}(\mathrm{pH} 7.4,10 \mathrm{mM})$ and $\mathrm{CH}_{3} \mathrm{CN}$ (98/2, vol\%). e, Partial ${ }^{1} \mathrm{H},{ }^{1} \mathrm{H}$ NOESY NMR spectrum $(850 \mathrm{MHz}, 343 \mathrm{~K})$ of $2(1 \mathrm{mg} / \mathrm{mL})$ in PB $(\mathrm{pH} 7.4,50 \mathrm{mM})$. Intermolecular NOE cross-peaks are circled in the NMR spectrum. f, Proposed molecular stacking mode of the Pt-tpy group in $\mathbf{2}_{\mathrm{NF}}$ based on the NOESY NMR study. Red arrows indicate intermolecular NOE interactions between protons. $\mathbf{g}$, Proposed arrangement of monomer $\mathbf{2}$ in $\mathbf{2}_{\mathrm{NF}}$ based on microscopy and NMR studies. The nanofibers showed a mean interfiber distance of $2.9 \mathrm{~nm}$. Nanofiber formation also promotes NIR emission originated from the Pt-tpy groups in $\mathbf{2}_{\mathrm{NF}}$. 
The growth of the nanofibers $\mathbf{2}_{\mathrm{NF}}$ was visualized in A549 lung alveolar adenocarcinoma cells and MDA-MB-231 metastatic breast cancer cells using confocal laser scanning microscopy (Fig. 4a, Supplementary Figs 46 and 47). Within 4 h, TAT-dependent internalization of 1-TAT $(25 \mu \mathrm{M})$ was observed (Fig. 4a). Assisted by the NIR emission of the platinum (II) center, correlative light-electron microscopy (CLEM) observed that newly formed luminescent nanofibers within the cytoplasm occur after escape from the endosomal vesicles (Fig. 4b, Supplementary Figs 48 and 49). In line with the $\mathrm{pH}$-dependence of endogenous $\mathrm{H}_{2} \mathrm{O}_{2}$ activity on the boronic acid, the TEM micrographs revealed that the entry into the near-neutral $(\mathrm{pH} \mathrm{7.4)}$ cytosol from the acidic $(\mathrm{pH} \mathrm{6.0)}$ endosomes initiates the nanofiber formation. At the onset of nanofiber growth, the first metabolic consequence of newly formed ADMI $\mathbf{2}_{\mathrm{NF}}$ can be detected through early changes in the cytoskeleton, whose formation is tightly coupled to glycolytic pathways ${ }^{30,31}$. An investigation using cytoskeleton stain Phalloidin-iFluor ${ }^{\mathrm{TM}}$ 405 demonstrated a restructuring of actin filaments towards the cellular membrane (Fig. 4c, Supplementary Fig 52). Using both $10 \mu \mathrm{M}$ and $25 \mu \mathrm{M}$, the appearance of fluorescent loci along the cell membrane was only observed at $25 \mu \mathrm{M}$ (Fig. 4c, lower panel), suggesting that a critical concentration threshold of $\mathbf{2}_{\mathrm{NF}}$ has to be reached for actin restructuring. 


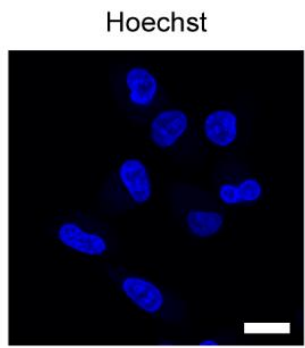

b

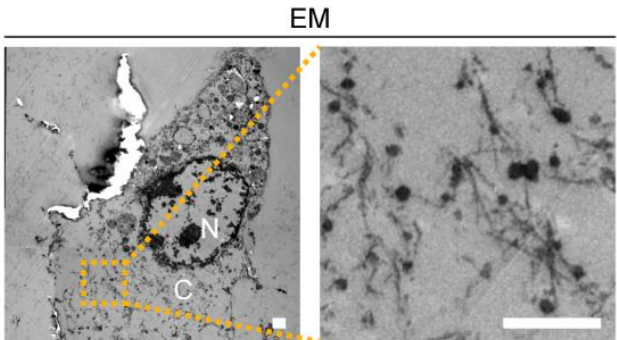

C
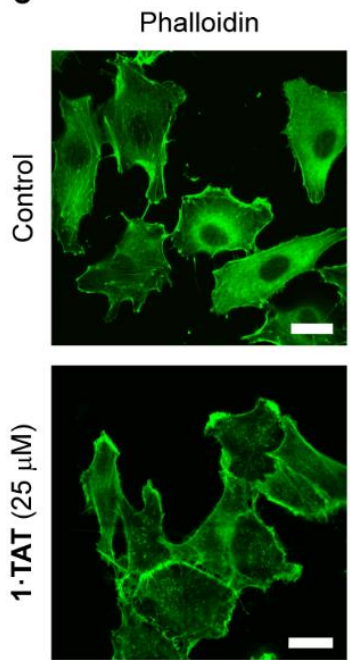

$2_{\mathrm{NF}}$
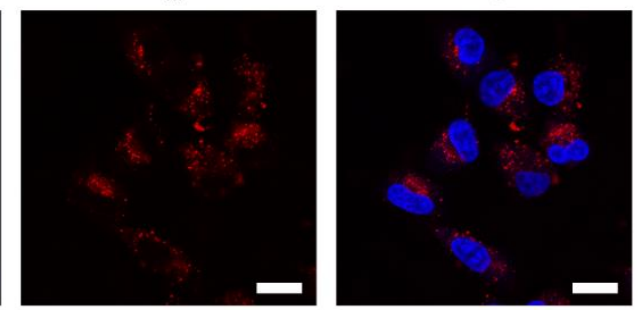

LM

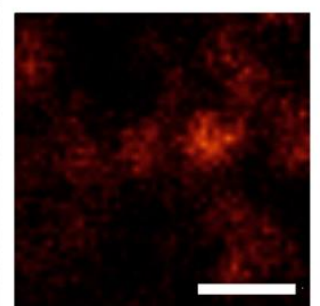

Merge
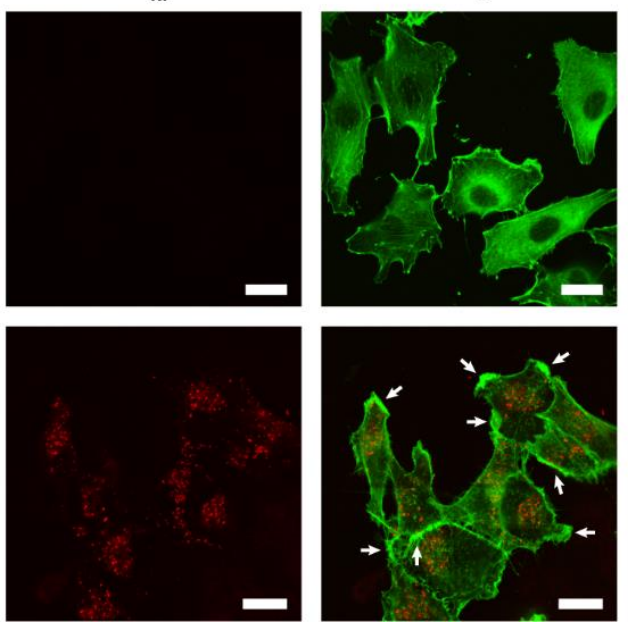

$\mathbf{2}_{\mathrm{NF}}$
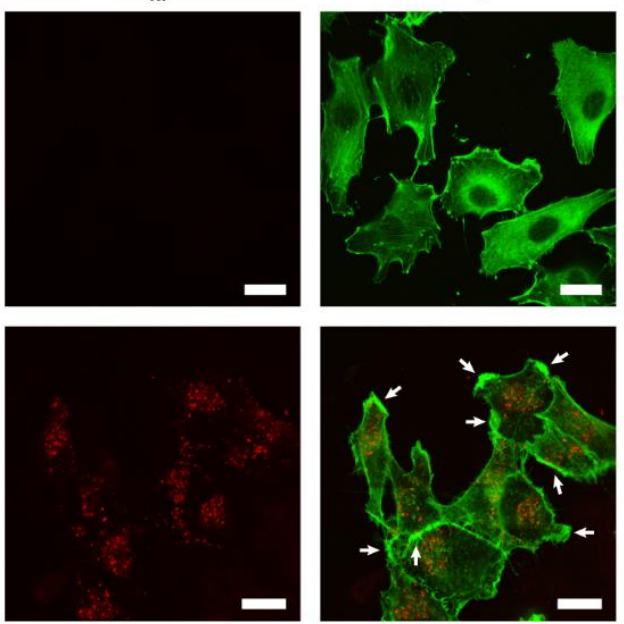

Brightfield

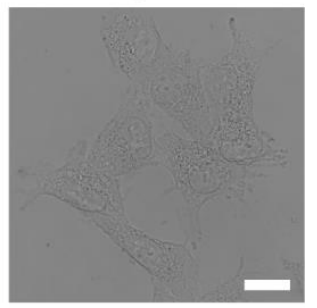

CLEM

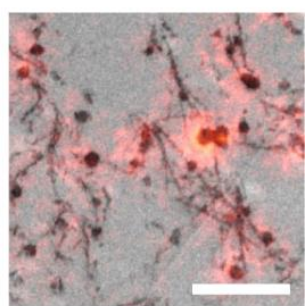

Brightfield
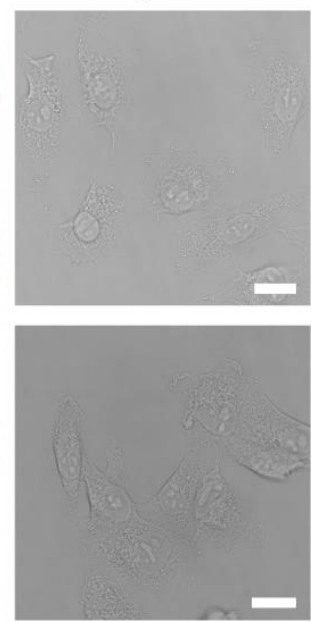

Fig. 4| Internalization of 1.TAT and assembly into ADMI nanofibers $\mathbf{2}_{\mathrm{NF}}$ in the cytoplasm that restructure actin filaments. a, Confocal laser scanning micrographs of A549 cells treated for $4 \mathrm{~h}$ with 1.TAT $(25 \mu \mathrm{M})$ and Hoechst 33342 dye. Scale bars, $20 \mu \mathrm{m}$. b, CLEM of A549 cells treated for $6 \mathrm{~h}$ with 1.TAT $(50 \mu \mathrm{M})$. N, nucleus. C, Cytosol. Scale bars, $1 \mu \mathrm{m}$. c, Confocal laser scanning micrographs of A549 cells treated for $4 \mathrm{~h}$ with 1.TAT $(25 \mu \mathrm{M})$ and Phalloidin-iFluor ${ }^{\mathrm{TM}} 405$. White arrows indicate the fluorescent loci along the cell membrane. Scale bars, $20 \mu \mathrm{m}$. 


\section{ADMI nanofibers $22_{\mathrm{NF}}$ inhibit AGlyc and OxPhos metabolic pathways}

We investigate the impact of the ADMI $\mathbf{2}_{\mathrm{NF}}$ on the main mechanisms of cellular metabolism, AGlyc and OxPhos pathways, which are core upstream processes involving at least $10 \%$ of the human proteome ${ }^{32}$. In cancer cells, metabolism is particularly altered to sustain perpetual growth, metastasis, and the unconventional use of glucose in the presence of oxygen ${ }^{16-19}$. Both the consumption of oxygen to fuel mainly OxPhos, and the extracellular acidification as a function of AGlyc were analyzed in A549 and MDA-MB-231 cells using a Seahorse ${ }^{\circledR}$ Extracellular Flux Analyzer (Fig. 5). Treatment of both cell lines with 1-TAT to form the ADMI $\mathbf{2}_{\mathrm{NF}}$ demonstrated a reduction in the oxygen consumption rate (OCR) within $4 \mathrm{~h}$ (Fig. $5 \mathrm{c}$ ), with $25 \mu \mathrm{M}$ or $10 \mu \mathrm{M}$ inducing respectively a 41 -fold and a 7 -fold decrease (Fig. 5d). The OCR, expressed as pmol/min, is a first measure of OxPhos (Fig. 5a, b) activity, which take place primarily in the mitochondria.

Key parameters of mitochondrial respiration were investigated to unravel more in detail the impact of the ADMI on OxPhos by sequential use of modulators of the electron transport chain (ETC): rotenone (Complex I), antimycin A (Complex III) and oligomycin (ATP synthase) and FCCP (Carbonyl cyanide 4-(trifluoromethoxy)phenylhydrazone) disrupting the mitochondrial membrane potential ${ }^{33,34}$. Treated cells exhibit less than $10 \%$ of the FCCP-induced maximal respiration compared to untreated cells, highlighting the inability of cells to address a challenging energy demand when stimulated to operate at maximum capacity. (Fig. 5e). Thus, in addition to the impact on OxPhos depicted by the acute response, the ADMI induces a massive and more stable effect on mitochondrial respiration that prevents cells from adapting to stress. This phenomenon is further confirmed by a reduction of most of the OxPhos-related parameters, such as ATP production, spare respiratory capacity, and proton leak, which is a potential indicator of mitochondrial damage (Supplementary Figs 55, 56). Based on the ADMI burden on mitochondrial respiration, we subsequently evaluated parameters related to AGlyc where cancer cells are known for being able to rewire their cell metabolism between OxPhos and AGlyc, upregulating accordingly one of the two metabolic pathways to meet their energetic needs.

Therefore, the AGlyc of A549 and MDA-MB-231 cells was measured using their extracellular acidification rate $\left(E C A R_{\text {glyc }}\right)$, expressed as $\mathrm{mpH} / \mathrm{min}$ (Fig. $\left.5 f-g\right)$. Extracellular acidification occurs when glucose is converted to pyruvate and lactate, accompanied by the extrusion of protons into extracellular space ${ }^{35}$ (Fig. 5f). The cells first underwent glucose starvation to account for the basal non-glycolytic acidification, followed by the addition of saturating amounts of glucose to trigger glycolysis. The formation of the nanofibers at $25 \mu \mathrm{M}$ shuts down glycolysis of A549 and MDA-MB-231 to $6 \pm 1 \%$ and $13 \pm 3 \%$, respectively (Fig. 5i). The capability of cancer cells rewiring their metabolism ${ }^{36}$ was demonstrated by adding oligomycin, the ATP synthase inhibitor ${ }^{33}$, to shut down OxPhos and thereby inducing the switch to glycolysis. In the untreated control cells, the incubation with oligomycin raised the glycolytic activity by $+11.5 \pm 4.0 \mathrm{mpH} / \mathrm{min}$ (A549) as well as $+8.2 \pm 3.5 \mathrm{mpH} / \mathrm{min}$ (MDAMB-231) (Fig. 5j). This adaptation is termed as the glycolytic reserve, which is activated upon mitochondrial stress/dysfunction.

In comparison to cells where the ADMI is formed, inhibition of OxPhos by oligomycin increased the glycolytic acidification by only $0.5 \mathrm{mpH} / \mathrm{min}$ for $\mathrm{A} 549$ cells and $0.2 \mathrm{mpH} / \mathrm{min}$ for MDA-MB-231 cells (Fig. 5h). Hence, even under severe stress conditions, aerobic glycolysis remained low, suggesting that the capabilities of the cells to compensate this inhibited pathway was limited. To further ascertain that the extracellular acidification is the consequence of glucose metabolism, 2-deoxy-glucose, a 
competitive inhibitor towards glucose hexokinase ${ }^{33}$ was added. By inhibiting the first enzyme of glycolysis and thus the suppression of ECAR, we demonstrated that the observed extracellular acidification is an accurate measure of their glycolytic activities. In summary, ADMI have shown the ability to impair cancer cell fitness by interfering with both OxPhos and AGlyc and preventing metabolic adaptation, one of the most characteristic features that cancer cells use to react and address stress stimuli ${ }^{37}$. In A549 cells, apoptotic resistance have been linked to the attenuation of OxPhos while increasing the rate of glycolysis, and that targeting OxPhos have led to reduced drug resistance ${ }^{17}$ and cancer proliferation ${ }^{38}$. Similarly, in MDA-MB-231, among the most aggressive cancer subtypes with the worst prognosis, the metabolic plasticity showed an even higher relevance in clinical outcomes where the targeting both OxPhos and AGlyc is the current state-of-the-art to control its progression $^{21,39,40}$. 

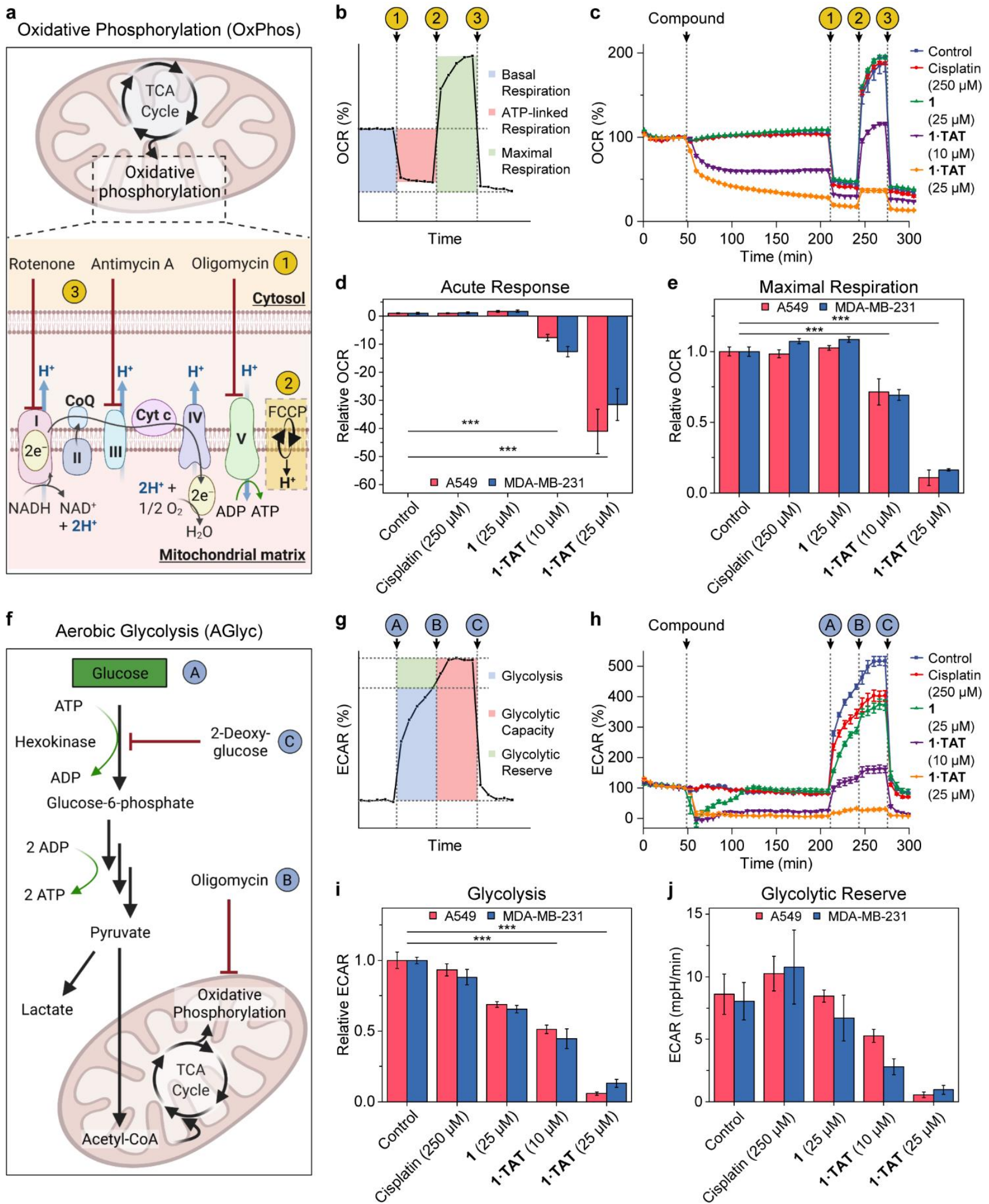

Fig. 5| ADMI 2NF inhibits both AGlyc and OxPhos metabolism of A549 and MDA-MB-231 cells. a, Scheme of a mitochondrion with focus on the electron transport chain, site of oxidative phosphorylation. The compounds of the Mito stress test modulate the ETC when added in the indicated injection sequence. $\mathbf{b}$, Profile of the oxygen consumption rate (OCR) during the Mito stress test. Numbers represent the compounds of the Mito stress test shown in Fig. 5a. c, Effect of the ADMI on the oxygen consumption rate of MDA-MB-231. The last measurement before treatment injection is set as $100 \%$. d, e, Acute response (d) and maximal respiration (e) 
of A549 and MDA-MB-231 cells. f, Schematic representation of glycolysis and cell respiration, which are affected by the compounds of the glycolysis stress test. Each target of the modulators of the glycolysis and oxidative phosphorylation used in the assay are indicated with respect to the injection sequence. $\mathbf{g}$, Profile of the extracellular acidification rate (ECAR) during the glycolysis stress test. Characters represent the compounds of the glycolysis stress test shown in Fig. $5 f . \mathbf{h}$, Effect of the ADMI on the extracellular acidification rate (ECAR) of MDA-MB-231. i,j Effect of the ADMI on the glycolysis (i) and glycolytic reserve (j) of A549 and MDA-MB-231 cells. For $\mathbf{d}, \mathbf{e}$, and $\mathbf{i}$ the data are expressed as relative values to untreated control cells. Data are presented as mean \pm s.e.m., $n>5$ independent experiments. Statistical significance was calculated by ANOVA with a Tukey post hoc test. ${ }^{* * *}: \mathrm{P}<0.001$.

\section{ADMI nanofibers $2 \mathrm{NF}$ induces early apoptosis}

With the ADMI showing inhibitory activities in both AGlyc and OxPhos processes, the production of metabolites such as glucose-6-phosphate (G6P) are negatively affected. Hence, G6P related pathways such as the downstream production of NADPH by glucose-6-phosphate dehydrogenase will be challenged, leading to the downregulation of histone deacetylase (HDAC) activity ${ }^{41}$. Indeed, ADMI treated A549 and MDA-MB-231 cells showed concentration dependent reduction in HDAC activity at $4 \mathrm{~h}$, with $25 \mu \mathrm{M}$ showing a 25\% and 10\% decrease, respectively (Supplementary Fig. 63). With the impairment of metabolic pathways and HDAC activity, apoptosis of the cells was evaluated using Annexin V-FITC and cell viability assays. At $25 \mu \mathrm{M}$, the ADMl induced the presence of phosphatidylserine on the external leaflet of the cell membrane and was detected by the binding of Annexin V-FITC. This characteristic observation of early apoptosis suggests that the population of cells were undergoing the hallmark changes associated with programmed cell death (Fig. 6a, Supplementary Fig.64) ${ }^{42}$. The $I_{50}$ value of the ADMI was found to be comparable for both A549 cells $(60.6 \mu \mathrm{M})$ and MDA-MB-231 cells $(58.5 \mu \mathrm{M})$ at $4 \mathrm{~h}$, while the cisplatin control requires $24 \mathrm{~h}$ to show an effect on cell viability (Fig. 6b, Supplementary Fig. 65). Cisplatin was used as a comparison and reference due to its use as a broad-spectrum chemotherapeutics for solid malignancies even though cisplatin resistance remained a widespread problem ${ }^{43}$. The disparity in time-dependent efficacies of cisplatin and the ADMI is apparent likewise in the metabolic studies, where a $4 \mathrm{~h}$ cisplatin treatment did not produce observable effects on AGlyc and OxPhos even at a 10-fold higher $(250 \mu \mathrm{M})$ concentration (Fig. 5). It is interesting to note that even though the formation of nanofibers demonstrates a more significant impact on oxygen-dependent ATP production on A549 cells compared to MDA-MB-231 cells, the $\mathrm{IC}_{50}$ value of the ADMI on both cell lines $(60.6 \mu \mathrm{M} / \mathrm{A} 549 ; 58.5$ $\mu \mathrm{M} / \mathrm{MDA}-\mathrm{MB}-231$ ) are not significantly different. We hypothesize that upon the critical assembly concentration of the nanofibers, the damage threshold towards cellular metabolism has reached a point of no return. At this stage, any further damage on cells that are already undergoing apoptosis would not provide additional statistical weight on the overall cell viability. 
a
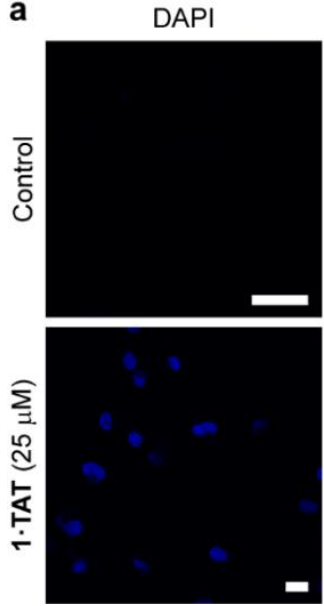

Annexin V-FITC
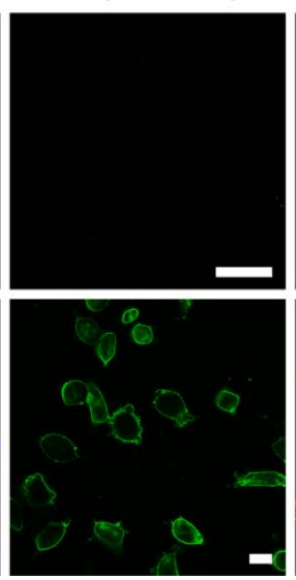
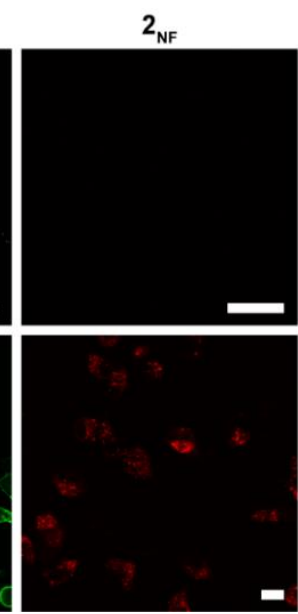

Merge Brightfield
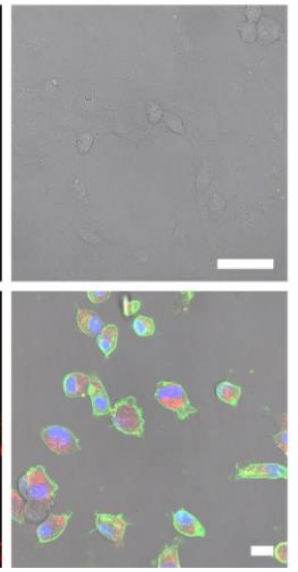

b

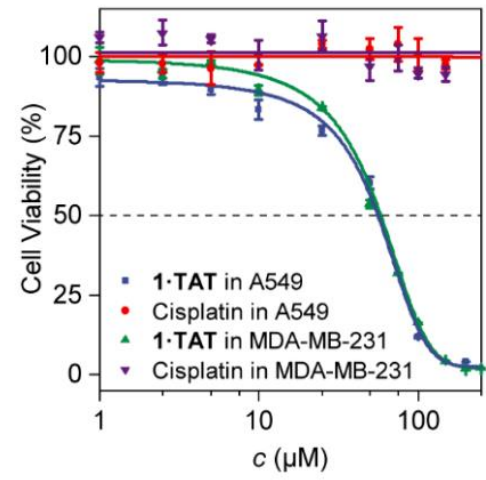

Fig. 6| Toxicity of 1-TAT towards A549 and MDA-MB-231 cells. a, The apoptotic nature of cell death was investigated for $A 549$ cells using Annexin $V$ assay. Cells were incubated $4 \mathrm{~h}$ with 1.TAT (red) and treated with Annexin V (green) afterwards. Nuclei were stained using DAPI (blue). Annexin V binds to the cell membrane upon an inversion of phosphatidylserine motifs towards the extracellular space, indicating apoptosis. Scale Bars, $50 \mu \mathrm{m}$. b, Cell viability assay of A549 and MDA-MB-231 cells treated with 1.TAT and cisplatin for $4 \mathrm{~h}$, revealing viable cells after cisplatin treatment and significantly reduced cell viability after application of the ADMI. The cell viability was determined using the CellTiter-Glo Luminescence Cell Viability Assay Kit.

\section{Conclusions}

In conclusion, we have designed a pro-assembling $\mathrm{Pt}(\mathrm{II})$-containing peptide that undergoes a stepwise transformation into a NIR emitting ADMI within A549 and MDA-MB-231 cells. The Pt-tpy complex directs the supramolecular order and directionality of the packing within the fiber axis, while providing NIR photoluminescence beyond the autofluorescence of the cells. The formation of the ADMI rapidly damages essential metabolic pathways, AGlyc and OxPhos, preventing the cells from mounting adaptive strategies that effectively resist specific small molecule inhibitors, increase aggressiveness and metastatic potential. Downstream pathways intrinsically linked to ATP production (cytoskeleton) and glucose metabolites (HDAC) are found to be impaired, thus confirming the mechanistic origin of the ADMI. Apoptosis is induced within $4 \mathrm{~h}$ compared with cisplatin (24 h) and found to leverage a similar impact even on different cell types. By demonstrating that complex cellular functions can be addressed purely by supramolecular order, we hope that synthetic nanostructure formation in cells can be established as an alternative therapeutic platform in the near future.

\section{References}

1. Pieters, B. J. G. E., van Eldijk, M. B., Nolte, R. J. M. \& Mecinović, J. Natural supramolecular protein assemblies. Chem. Soc. Rev. 45, 24-39 (2016).

2. McGlinchey, R. P. et al. The repeat domain of the melanosome fibril protein Pmel17 forms the amyloid core promoting melanin synthesis. Proc. Natl. Acad. Sci. USA 106, 13731-13736 (2009).

3. Dean, D. N. \& Lee, J. C. Defining an amyloid link Between Parkinson's disease and melanoma. Proc. Natl. Acad. Sci. USA 117, 22671-22673 (2020). 
4. Westermark, P., Andersson, A. \& Westermark, G. T. Islet Amyloid Polypeptide, Islet Amyloid, and Diabetes Mellitus. Physiol. Rev. 91, 795-826 (2011).

5. Iadanza, M. G., Jackson, M. P., Hewitt, E. W., Ranson, N. A. \& Radford, S. E. A new era for understanding amyloid structures and disease. Nat. Rev. Mol. Cell Biol. 19, 755-773 (2018).

6. Hendricks, M. P., Sato, K., Palmer, L. C. \& Stupp, S. I. Supramolecular Assembly of Peptide Amphiphiles. Acc. Chem. Res. 50, 2440-2448 (2017).

7. Goor, O. J. G. M., Hendrikse, S. I. S., Dankers, P. Y. W. \& Meijer, E. W. From supramolecular polymers to multi-component biomaterials. Chem. Soc. Rev. 46, 6621-6637 (2017).

8. Qi, G.-B., Gao, Y.-J., Wang, L. \& Wang, H. Self-Assembled Peptide-Based Nanomaterials for Biomedical Imaging and Therapy. Adv. Mater. 30, 1703444 (2018).

9. Shy, A. N., Kim, B. J. \& Xu, B. Enzymatic Noncovalent Synthesis of Supramolecular Soft Matter for Biomedical Applications. Matter 1, 1127-1147 (2019).

10. Yuan, Y. et al. Furin-mediated intracellular self-assembly of olsalazine nanoparticles for enhanced magnetic resonance imaging and tumour therapy. Nat. Mater. 18, 1376-1383 (2019).

11. Pieszka, M. et al. Controlled Supramolecular Assembly Inside Living Cells by Sequential Multistaged Chemical Reactions. J. Am. Chem. Soc. 142, 15780-15789 (2020).

12. Yang, L. et al. Desuccinylation-Triggered Peptide Self-Assembly: Live Cell Imaging of SIRT5 Activity and Mitochondrial Activity Modulation. J. Am. Chem. Soc. 142, 18150-18159 (2020).

13. Miki, T. et al. Intracellular artificial supramolecules based on de novo designed $\mathrm{Y} 15$ peptides. Nat. Commun. 12 (2021).

14. Knauer, M. F., Soreghan, B., Burdick, D., Kosmoski, J. \& Glabe, C. G. Intracellular accumulation and resistance to degradation of the Alzheimer amyloid A4/beta protein. Proc. Natl. Acad. Sci. USA 89, 74377441 (1992).

15. Yuan, Y. et al. Intracellular Self-Assembly of Taxol Nanoparticles for Overcoming Multidrug Resistance. Angew. Chem. Int. Ed. 54, 9700-9704 (2015).

16. Zhu, J. \& Thompson, C. B. Metabolic regulation of cell growth and proliferation. Nat. Rev. Mol. Cell Biol. 20, 436-450 (2019).

17. Vasan, K., Werner, M. \& Chandel, N. S. Mitochondrial Metabolism as a Target for Cancer Therapy. Cell Metab. 32, 341-352 (2020).

18. Martínez-Reyes, I. \& Chandel, N. S. Cancer metabolism: looking forward. Nat. Rev. Cancer 21, 669-680 (2021).

19. DeBerardinis, R. J. \& Chandel, N. S. We need to talk about the Warburg effect. Nat. Metab. 2, 127-129 (2020).

20. Cheng, G. et al. Targeting lonidamine to mitochondria mitigates lung tumorigenesis and brain metastasis. Nat. Commun. 10 (2019).

21. Fiorillo, M., Scatena, C., Naccarato, A. G., Sotgia, F. \& Lisanti, M. P. Bedaquiline, an FDA-approved drug, inhibits mitochondrial ATP production and metastasis in vivo, by targeting the gamma subunit (ATP5F1C) of the ATP synthase. Cell Death Differ. 28, 2797-2817 (2021).

22. Icard, P. et al. How the Warburg effect supports aggressiveness and drug resistance of cancer cells? Drug Resist. Updat. 38, 1-11 (2018).

23. Yam, V. W.-W., Chan, A. K.-W. \& Hong, E. Y.-H. Charge-transfer processes in metal complexes enable luminescence and memory functions. Nat. Rev. Chem. 4, 528-541 (2020).

24. Aliprandi, A., Mauro, M. \& De Cola, L. Controlling and imaging biomimetic self-assembly. Nature Chemistry 8, 10-15 (2015).

25. Herce, H. D. \& Garcia, A. E. Molecular dynamics simulations suggest a mechanism for translocation of the HIV-1 TAT peptide across lipid membranes. Proc. Natl. Acad. Sci. USA 104, 20805-20810 (2007).

26. Ng, D. Y. W. et al. Constructing Hybrid Protein Zymogens through Protective Dendritic Assembly. Angew. Chem. Int. Ed. 53, 324-328 (2014). 
27. Wong, V. C.-H. et al. Formation of $1 \mathrm{D}$ Infinite Chains Directed by Metal-Metal and/or $\pi-\pi$ Stacking Interactions of Water-Soluble Platinum(II) 2,6-Bis(benzimidazol-2'-yl)pyridine Double Complex Salts. J. Am. Chem. Soc. 140, 657-666 (2018).

28. Fleming, S. \& Ulijn, R. V. Design of nanostructures based on aromatic peptide amphiphiles. Chem. Soc. Rev. 43, 8150-8177 (2014).

29. Ranjbar, B. \& Gill, P. Circular Dichroism Techniques: Biomolecular and Nanostructural Analyses- A Review. Chem. Biol. Drug Des 74, 101-120 (2009).

30. $\mathrm{Hu}, \mathrm{H}$. et al. Phosphoinositide 3-Kinase Regulates Glycolysis through Mobilization of Aldolase from the Actin Cytoskeleton. Cell 164, 433-446 (2016).

31. Park, J. S. et al. Mechanical regulation of glycolysis via cytoskeleton architecture. Nature 578, 621-626 (2020).

32. Romero, P. et al. Computational prediction of human metabolic pathways from the complete human genome. Genome Biol. 6 (2004).

33. Divakaruni, A. S., Paradyse, A., Ferrick, D. A., Murphy, A. N. \& Jastroch, M. Analysis and Interpretation of Microplate-Based Oxygen Consumption and pH Data. Methods Enzymol., 309-354 (2014).

34. Trotta, A. P. et al. Disruption of mitochondrial electron transport chain function potentiates the pro-apoptotic effects of MAPK inhibition. J. Biol. Chem. 292, 11727-11739 (2017).

35. Ippolito, L., Morandi, A., Giannoni, E. \& Chiarugi, P. Lactate: A Metabolic Driver in the Tumour Landscape. Trends Biochem. Sci. 44, 153-166 (2019).

36. Shiratori, R. et al. Glycolytic suppression dramatically changes the intracellular metabolic profile of multiple cancer cell lines in a mitochondrial metabolism-dependent manner. Sci. Rep. 9 (2019).

37. Hanahan, D. \& Weinberg, Robert A. Hallmarks of Cancer: The Next Generation. Cell 144, 646-674 (2011).

38. Rao, S. et al. AlF-regulated oxidative phosphorylation supports lung cancer development. Cell Res. 29, 579-591 (2019).

39. Lanning, N. J. et al. Metabolic profiling of triple-negative breast cancer cells reveals metabolic vulnerabilities. Cancer Metab. 5 (2017).

40. Jia, D. et al. Elucidating cancer metabolic plasticity by coupling gene regulation with metabolic pathways. Proc. Natl. Acad. Sci. USA 116, 3909-3918 (2019).

41. Vogelauer, M., Krall, A. S., McBrian, M. A., Li, J.-Y. \& Kurdistani, S. K. Stimulation of Histone Deacetylase Activity by Metabolites of Intermediary Metabolism. J. Biol. Chem. 287, 32006-32016 (2012).

42. Demchenko, A. P. Beyond annexin V: fluorescence response of cellular membranes to apoptosis. Cytotechnology 65, 157-172 (2012).

43. Shen, D.-W., Pouliot, L. M., Hall, M. D., Gottesman, M. M. \& Sibley, D. R. Cisplatin Resistance: A Cellular Self-Defense Mechanism Resulting from Multiple Epigenetic and Genetic Changes. Pharmacol. Rev. 64, 706-721 (2012). 


\section{Methods}

Synthesis and characterization of the complexes. Solid-phase peptide synthesis was conducted to prepare the alkynyl-functionalized peptidic precursors. Dehydrohalogenation reaction between the alkynyl group and a chloroplatinum (II) complex under an inert atmosphere led to the coordination of the alkynyl ligand to the Pt-tpy moiety to afford 1, the precursor for 1-TAT with a free boronic acid group. Linear conjugate 2 was synthesized, as a control, using a similar strategy. Complexation of $\mathbf{1}$ and the SHA-functionalized TAT peptide motif via SHAboronic acid dynamic covalent bond was achieved by mixing them in a 1:1 molar ratio in a mixture of DMSO and phosphate buffer (PB, pH 7.4, $50 \mathrm{mM}$ ) (DMSO/PB, 2/98, vol\%), leading to the formation of 1.TAT. The compounds were identified by NMR spectroscopy, high-performance liquid chromatography, and mass spectrometry. A detailed procedure for the synthesis and characterization of the complexes can be found in the Supplementary Information.

LC-MS analysis of $\mathrm{H}_{2} \mathrm{O}_{2}$-induced conversion. Complex 1 was dissolved in a mixture of $\mathrm{NH}_{4} \mathrm{HCO}_{3}$ buffer $(\mathrm{pH}$ 7.4, $20 \mathrm{mM})$ and $\mathrm{CH}_{3} \mathrm{OH}\left(90 / 10\right.$, vol\%) at a concentration of $100 \mu \mathrm{M}$. Equal volume of $\mathrm{H}_{2} \mathrm{O}_{2}(1 \mathrm{mM})$ solution in a mixture of $\mathrm{NH}_{4} \mathrm{HCO}_{3}$ buffer $(\mathrm{pH} 7.4,20 \mathrm{mM})$ and $\mathrm{CH}_{3} \mathrm{OH}(90 / 10$, vol\%) was added to initiate the conversion. LC-MS analysis of the mixture was performed on a Shimadzu LC-MS 2020 equipped with a Kinetex EVO C18 column ( $50 \times 2.1 \mathrm{~mm}, 2.6 \mu \mathrm{m}, 100 \AA)$, an SPD-20A UV-Vis detector, and an electrospray ionization source. MilliQ water, acidified with $0.1 \%$ formic acid, and $\mathrm{CH}_{3} \mathrm{CN}$ were used as solvents for all measurements. The solvent gradient started with $5 \% \mathrm{CH}_{3} \mathrm{CN}$ and $95 \%$ water. This solvent ratio was kept constant for 2 min, and then the $\mathrm{CH}_{3} \mathrm{CN}$ content was linearly increased to $95 \%$ over $14 \mathrm{~min}$. The molar ratio of the compounds was calculated using peak areas at $254 \mathrm{~nm}$.

Self-assembly formation of $\mathbf{2}_{\mathrm{NF}}$. Monomeric complex 2 was dissolved in DMSO at a high concentration (10 $\mathrm{mM}$ ) before diluted to different concentrations using $\mathrm{PB}(\mathrm{pH} 7.4,50 \mathrm{mM})$. Self-assembly was induced by agitating the solution in an Eppendorf Thermomixer for $2 \mathrm{~h}$ at $37^{\circ} \mathrm{C}$ at 500 r.p.m.

TEM analysis. TEM samples were prepared by dropping a solution of $\mathbf{2} \mathrm{NF}$ onto a carbon-coated copper grid. After $5 \mathrm{~min}$, the excess solution was removed by a filter paper. The sample was then stained inverting the TEM grid (sample side down) on a droplet $(10 \mu \mathrm{L})$ of $2 \%$ uranyl acetate solution for 30 seconds. After removing the staining solution with a filter paper, the sample was washed with Milli-Q water for three times (shaken in water for 6 seconds for each time). After drying in air, the measurement was conducted on a JEOL JEM-1400 TEM operating at an accelerating voltage of $120 \mathrm{kV}$.

SAED Analysis. Sample for SAED analysis was prepared by drop-casting a solution of $\mathbf{2}_{\mathrm{NF}}$ in $\mathrm{H}_{2} \mathrm{O}$ on an ultrathin carbon grid. SAED analysis was performed on an image-side aberration-corrected FEI Titan 80-300 operated at $300 \mathrm{kV}$. The microscope is equipped with a CEOS hexapole aberration-corrector which corrects the geometrical axial aberrations up to the $3^{\text {rd }}$-order. Diffraction data acquisition was conducted on a Gatan UltraScan CCD camera. For SAED acquisition, we used a selected-area aperture with a physical diameter of $50 \mu \mathrm{m}$, corresponding to a diameter of $700 \mathrm{~nm}$ in the image plane. In order to avoid substantial electron radiation damage on the specimen, a low-dose technique has been applied. The total electron dose for SAED acquisition was $0.8 \mathrm{e}-/ \AA^{2}$ (dose rate: $0.04 \mathrm{e}-/ \AA^{2} \mathrm{~s}$, acquisition time: $20 \mathrm{~s}$ ).

Cryo-TEM analysis. For cryo-TEM examinations the sample was prepared using a Mark V Vitrobot $\circledast$ (Thermo Fisher Scientific) cryo plunge device. Samples were plunged from a climate chamber operated at room temperature with $100 \%$ relative humidity. The sample dispersion was applied either to a Quantifoil @ or lacey holey carbon grid and plunged into liquid ethane. Subsequently, the specimen grid was transferred to a Titan Krios G4 TEM (Thermo Fisher Scientific). Images were acquired using a Gatan K3 direct detection camera.

${ }^{1} \mathrm{H},{ }^{1} \mathrm{H}$ NOESY NMR experiments. The NOESY NMR measurements were performed with a $5 \mathrm{~mm}$ QXI ${ }^{1} \mathrm{H} /{ }^{13} \mathrm{C} /{ }^{15} \mathrm{~N} /{ }^{31} \mathrm{P}$ probe equipped with a z-gradient on the $850 \mathrm{MHz}$ Bruker AVANCE III system. The spectroscopic 
widths of the ${ }^{1} \mathrm{H},{ }^{1} \mathrm{H}$ NOESY experiments were $13500 \mathrm{~Hz}(850 \mathrm{MHz} ; 16 \mathrm{ppm})$ in both dimension (f1 and f2) and the relaxation delay is $2 \mathrm{~s}$. The mixing time was kept at $300 \mathrm{~ms}$. A detailed analysis for the NOESY NMR experiments can be found in the Supplementary Information.

Cell culture. A549 and MDA-MB-231 cells were cultured at $37{ }^{\circ} \mathrm{C}$ and $5 \% \mathrm{CO}_{2}$ in Dulbecco's Modified Eagle's Medium (DMEM, high glucose), supplemented with 10\% FBS. Both cell lines were cultured in T75 culture flask and subcultivated two to three times per week.

Cellular uptake studies. A549 and MDA-MB-231 cells were seeded at a density of 25,000 cells/well in an 8well confocal plate. After adhering for $24 \mathrm{~h}$, cells were treated with the sample for $4 \mathrm{~h}$ at $37^{\circ} \mathrm{C}$. Before adding to the cells, samples were pre-incubated to form the 1-TAT by dissolving 1 in DMSO and mixing with an equimolar amount of the TAT peptide, which was dissolved in Dulbecco's PBS. The sample was further diluted with DPBS to a final volume of $40 \mu \mathrm{L}$ (DMSO/DPBS, 10/90, vol\%). Sample solutions were further diluted 1:4 with DMEM and added to the cells (total DMSO content $=2 \%$ ), after removing the existing medium from the wells. After the incubation time was over, the cell nucleus was stained with Hoechst 33342 dye for 20 min at $37^{\circ} \mathrm{C}$. A459 cells were additionally treated with Phalloidin-iFluor ${ }^{\mathrm{TM}} 405$ instead of the Hoechst 33342 dye to stain the cytoskeleton. The staining solution was removed, and fresh FluoroBrite DMEM Medium was added to the cells before they were imaged by confocal laser scanning microscopy. Confocal images of cells were taken on a Leica TCS SP5 and Visitron Spinning Disc microscope. To monitor the Hoechst, DAPI and Phalloidin dyes, a $405 \mathrm{~nm}$ excitation diode laser was used with an emission filter from 415-500 nm. For Annexin-FITC, an Argon laser was used for excitation at $488 \mathrm{~nm}$ with an emission filter from 500-600 nm. To study the uptake of the compound 1.TAT and subsequent formation of $2 \mathbf{N F}$, a DPSS laser was used at $561 \mathrm{~nm}$ excitation and an emission range from 600-800 $\mathrm{nm}$.

CLEM studies. One day before treatment, cells were seeded and grown on carbon pre-coated sapphire disks ( $3 \mathrm{~mm}$; M. Wohlwend $\mathrm{GmbH}$ ), which were sterilized and oxygen plasma cleaned before use (30 s, $0.2 \mathrm{mbar}$, $20 \%$ power; Femto, diener electronic). The disks were placed in a 24-well plate with 50,000 cells/well, which was incubated at $37^{\circ} \mathrm{C}, 5 \% \mathrm{CO}_{2}$ overnight. After incubation with 1.TAT $(50 \mu \mathrm{M}, 6 \mathrm{~h})$, each sapphire disk was collected and slightly immersed into 1-hexadecene before placing them between two aluminum plates ( $3 \mathrm{~mm}$, Plano). The aluminum plates with the sample were placed into a specimen holder for high pressure freezing in a Wohlwend HPF Compact 01 high-pressure freezer with a pressure of 2100 bar for $2-3 \mathrm{~s}$. The specimen holder was withdrawn from the freezer and immersed into liquid nitrogen to release the sample. The frozen sample was then stored in a container filled with liquid nitrogen. Subsequently, freeze substitution of the sample was carried out in a $0.5 \mathrm{~mL}$ Eppendorf tube using an AFS2 freeze substitution unit (Leica). Each tube contained freeze substitution solution, consisting of $0.2 \mathrm{wt} / \mathrm{vol} \%$ osmium tetroxide, $0.1 \mathrm{wt} / \mathrm{vol} \%$ uranyl acetate, and $5 \%$ distilled water in acetone. The tubes were firstly kept at $-90^{\circ} \mathrm{C}$ and automatically warmed up to $0{ }^{\circ} \mathrm{C}$ in $24 \mathrm{~h}$. After keeping at room temperature for $1 \mathrm{~h}$, the substitution solution was removed, and the samples were washed three times with acetone. Each sample was infiltrated in an ascending epoxy resin series $(30 \%, 50 \%$, and $75 \%$ in acetone) for $1 \mathrm{~h}$ before final infiltration in 100\% epoxy resin overnight. Subsequently, each sample was transferred into a new Eppendorf tube containing freshly prepared pure epoxy resin for polymerization at $60^{\circ} \mathrm{C}$ for $72 \mathrm{~h}$. After polymerization, sample blocks were kept at room temperature until their sectioning. Sample blocks for each time point were trimmed and sectioned into $100 \mathrm{~nm}$ sections by a $35^{\circ}$ diamond knife (Diatome) in EM UC6 ultramicrotome (Leica). Sections were then carefully placed onto $\mathrm{H} 6$ copper finder grids (Plano) and imaged on a confocal light microscope (Leica SP5) using $561 \mathrm{~nm}$ excitation and a 610/60 ET bandpass. For later registration to TEM images, transmitted light and reflected light images were simultaneously recorded. Electron microscopy images were collected on a Jeol 1400 120kV using a 2K bottom mount CCD camera and a Tecnai F20 200 kV TEM by FEl using a K2 Gatan camera. The TEM images were stitched to show the entire cell. Registration of light and electron microscopy images was done by manual landmark registration on entire cells using ImageJ. Landmark selection was based on features identified in the reflected light and transmitted light images. 
Cell metabolism assay. The effect of the compounds on the cell metabolism of A549 and MDA-MB-231 was investigated using the Agilent Seahorse XFe96 Analyzer. Cells were seeded first in DMEM one day before the assay and incubated overnight at $37^{\circ} \mathrm{C}, 5 \% \mathrm{CO}_{2}$. The cartridge for sample loading was loaded with calibration solution $\left(200 \mu \mathrm{L}\right.$ per well) and incubated at $37^{\circ} \mathrm{C}$ in a non- $\mathrm{CO}_{2}$ incubator. On the day of the assay, XF DMEM, $\mathrm{pH} 7.4$ was supplemented with supplements according to the specific assay kit. Cells were washed once with XF DMEM $(200 \mu \mathrm{L})$ and the medium was substituted with fresh XF DMEM $(180 \mu \mathrm{L})$ and placed in a $37^{\circ} \mathrm{C}$ non$\mathrm{CO}_{2}$ incubator for 45 to 60 minutes. The cartridge was prepared by loading the samples $(20 \mu \mathrm{L})$ in Port $\mathrm{A}$ of each well and the components of the performed assay in Ports B-D. The cartridge and 96-well plate with the cells were loaded in the instrument and the content of the ports was injected sequentially according to the specific assay. The $\mathrm{pH}$, to monitor the extracellular acidification rate (ECAR) and the oxygen concentration, or to monitor the oxygen consumption rate (OCR) were constantly measured throughout the entire time of the assay.

For the Mito stress assay, ports B-D were loaded with Oligomycin (22 $\mu \mathrm{L})$, Carbonyl cyanide-4 (trifluoromethoxy) phenylhydrazone (FCCP) $(25 \mu \mathrm{L})$, and a mixture of Rotenone and Antimycin $\mathrm{A}(\operatorname{Rot} / \mathrm{AA})(27 \mu \mathrm{L})$. After loading the cell plate eight measurement cycles were performed as baseline control. After addition of the compound, twenty-five measurement cycles were performed before the components of the Mito stress assay were added. For each component five measurement cycles were performed.

Fir the glycolysis stress assay, cells were maintained for $1 \mathrm{~h}$ in Seahorse DMEM supplemented only with glutamine before the assay. Ports B-D were loaded with glucose $(22 \mu \mathrm{L})$, Oligomycin $(25 \mu \mathrm{L})$, and 2Deoxyglucose $(27 \mu \mathrm{L})$. After loading the cell plate eight measurement cycles were performed as baseline control. After addition of the compound twenty-five measurement cycles were performed before the components of the glycolysis stress assay were added. For each component five measurement cycles were performed.

Cell viability assay. A549 and MDA-MB-231 cells were both seeded at a density of 2,500 cells/well in a white half area 96-well plate and incubated at $37^{\circ} \mathrm{C}$ overnight to allow them to adhere. On the next day, different concentrations of the 1.TAT or cisplatin were prepared in PBS at a 10x concentration of the final concentration, and this solution $(20 \mu \mathrm{L})$ was added to a fresh medium $(180 \mu \mathrm{L})$. The old medium of the cells was removed and substituted with a fresh medium $(50 \mu \mathrm{L})$ containing different concentrations of sample, prepared as described before. For each condition, triplicates were performed. Cells were incubated with the sample for $4 \mathrm{~h}$ or $24 \mathrm{~h}$. After the treatment, CellTiter-Glo ${ }^{\circledR}$ Assay solution ( $50 \mu \mathrm{L}$ ) was added to each well, and the plate was placed on an orbital shaker for 2 minutes and subsequently incubated 10 minutes at room temperature. Luminescence was measured using a Promega GloMax ${ }^{\circledR}$-Multi Detection System using manufacturer's protocol.

\section{Acknowledgements}

The authors gratefully acknowledge financial support from the Max Planck-Bristol Centre for Minimal Biology. Z. Z. is supported by the Alexander von Humboldt Foundation. P.M. acknowledge the support from SFB 1066 B16 (Grant No 213555243). D.M., H.Q. and U.K. gratefully acknowledge the funding from the Germany Research Foundation (DFG) in SFB-1415 (Grant No. 417590517) and from the European Union's Horizon2020 research and innovation programme under Grant Agreement No. 881603 (GrapheneCore3).

\section{Author contributions}

Z.Z., D.Y.W.N and T.W. conceived and designed experiments. Z.Z. synthesized the compounds, conducted the kinetic and self-assembly analysis. K.M. and D.Y.W.N. performed the cellular studies. K.M. and P.M. conducted the cellular metabolism assay. S.X., Y.W., and C.J.W. assisted with microscopy analysis. L.X. assisted with 
chemical synthesis. A.K., S.H., and K.L. conducted the CLEM analysis. D.N., H.Q., and U.K. performed the selected-area electron diffraction analysis. M.W. provided the NMR expertise. I.L. and K.L. conducted the cryoTEM analysis. Z.Z., K.M., and D.Y.W.N. wrote the manuscript. D.Y.W.N. and T.W. supervised the project and corrected the manuscript. All authors read and approved the final manuscript. 